\title{
Elastic waves along a cylindrical borehole in a poroelastic medium saturated by two immiscible fluids
}

\author{
Ashish ARORA ${ }^{1}$ and S K TOMAR ${ }^{2}$ \\ ${ }^{1}$ Department of Mathematics, Guru Nanak Dev University, Amritsar 143 006, Punjab, India. \\ e-mail: aroraashish_a@rediffmail.com \\ ${ }^{2}$ Department of Mathematics, Panjab University, Chandigarh 160 014, India. \\ e-mail: sktomar@yahoo.com
}

\begin{abstract}
The propagation of elastic waves along a cylindrical borehole filled with/without liquid and embedded in an infinite porous medium saturated by two immiscible fluids has been studied. The theory of porous media saturated by two immiscible fluids developed by Tuncay and Corapcioglu (1997) is employed. Frequency equations determining the phase velocity of axial symmetric waves are obtained. It is found that the surface waves along cylindrical borehole are dispersive. The dispersion equation of Rayleigh-type surface waves along the boundary of a poroelastic solid half-space saturated by two immiscible fluids is also obtained. Some special cases have been deduced and the dispersion curves are obtained numerically for a peculiar model. It is found that the density of fluids affects the Rayleigh mode.
\end{abstract}

\section{Introduction}

The problem of propagation of waves along a cylindrical hole embedded in an infinite elastic medium is of great importance due to its manifold applications. In practice, the cylindrical hole may be realized by a bore hole or a mine gallery. Bore hole studies are of great help in exploration seismology, e.g., in exploration of oils, gases and hydro-carbons, etc. In the oil industry, acoustic borehole logging is commonly practiced. A borehole is drilled in a potential hydro-carbon reservoir and then probed with an acoustic tool. Biot (1952) was perhaps the first who studied the propagation of elastic waves in a cylindrical bore filled with and without fluid and embedded in an elastic solid of infinite extent. He treated the problem in two dimensions only and calculated the dispersion curves. Stilke (1959) obtained the solutions for the propagation of elastic waves at the surface of a tunnel-like hole with a circular border embedded in a three dimensional perfectly elastic, homogeneous and isotropic medium. He found that the phase and group velocities depend on the ratio between the wavelength and the circumference of the cylindrical hole. White (1962) discussed axially symmetric solutions for waves propagating along a cylinder in an infinite elastic solid. He presented the solutions describing the unattenuated wave propagation along the axis at phase velocities higher than the shear and compressional speeds in the solid. Special attention is given to the limiting case of phase velocity equal to compressional speed in the solid, which at low frequencies very closely approximates the coupling of a fluid-filled borehole to a plane compressional wave in the surrounding solid. Rosenbaum (1974) studied numerically the propagation of sound pulses along a cylindrical borehole and along a plane interface in a porous medium saturated by a single fluid. He found that permeability of the porous medium affects the attenuation of the signal only at high frequencies. Cheng et al (1987) studied the propagation of Stoneley waves in a cylindrical borehole. They found that the phase velocity and attenuation increase with permeability and porosity, but decrease with

Keywords. Borehole; wave propagation; frequency equation; phase velocity; group velocity; surface waves. 
increasing frequency. Sharma and Gogna (1990) studied the propagation of elastic waves in a cylindrical bore through a liquid saturated porous solid of infinite extent. They employed Biot's equations to derive the velocity dispersion equation appropriate to the surface wave propagating in a liquid filled porous solid containing empty/liquid filled borehole. Cheng and Toksoz (1981); Paillet and White (1982); Kurkjian and Chang (1986); Paillet and Cheng (1986); Tomar and Kumar (1999); Rama Rao and Vandiver (1999); Berger et al (2000) also attempted the problems of wave propagation in boreholes. Recently, Vashishth and Khurana (2005) studied the propagation of wave along a cylindrical borehole embedded in an anisotropic porous solid saturated by a viscous fluid. They used Biot's theory to derive the Christoffel equation for the propagation of elastic waves and studied the effects of transverse isotropy on the phase velocity dispersion curves of surface waves. Whether the anisotropy factor increases or borehole is filled with liquid, the phase velocity of the surface waves is found to increase.

In the present formulation, a cylindrical borehole embedded in a porous elastic medium saturated by two immiscible fluids is considered. Using the theory of Tuncay and Corapcioglu (1997) for poroelastic solid saturated by two immiscible fluids, we have studied the propagation of surface waves along the surface of a circular borehole, when it is empty and when it is filled with a fluid. Dispersion equations are derived in both cases. These frequency equations converge to Rayleigh wave dispersion equation in a limiting case. Numerical computation is also performed to find out the dispersion curves of surface waves and the velocity of Rayleigh waves.

\section{Elastodynamical equations and their solutions}

The differential equations governing the small displacement of solid and interstitial fluids, in the absence of body forces, in a porous elastic medium saturated by two immiscible fluids are given by (Tuncay and Corapcioglu 1997)

$$
\begin{aligned}
\left\langle\rho_{s}\right\rangle \frac{\partial^{2} \mathbf{u}_{s}}{\partial t^{2}}= & \nabla\left(\left(a_{11}+\frac{1}{3} G_{f r}\right) \nabla \cdot \mathbf{u}_{s}+a_{12} \nabla \cdot \mathbf{u}_{1}\right. \\
& \left.+a_{13} \nabla \cdot \mathbf{u}_{2}\right)+\nabla \cdot\left(G_{f r} \nabla \mathbf{u}_{s}\right) \\
& +c_{1}\left(v_{1}-v_{s}\right)+c_{2}\left(v_{2}-v_{s}\right)
\end{aligned}
$$

$$
\begin{aligned}
\left\langle\rho_{1}\right\rangle \frac{\partial^{2} \mathbf{u}_{1}}{\partial t^{2}}= & \nabla\left(a_{21} \nabla \cdot \mathbf{u}_{s}+a_{22} \nabla \cdot \mathbf{u}_{1}+a_{23} \nabla \cdot \mathbf{u}_{2}\right) \\
& -c_{1}\left(v_{1}-v_{s}\right), \\
\left\langle\rho_{2}\right\rangle \frac{\partial^{2} \mathbf{u}_{2}}{\partial t^{2}}= & \nabla\left(a_{31} \nabla \cdot \mathbf{u}_{s}+a_{32} \nabla \cdot \mathbf{u}_{1}+a_{33} \nabla \cdot \mathbf{u}_{2}\right) \\
& -c_{2}\left(v_{2}-v_{s}\right),
\end{aligned}
$$

where

$$
\begin{aligned}
& a_{11}=K_{f r}, \quad a_{12}=a_{21}=K_{1} \alpha_{s} S_{1}\left(A_{2}+K_{2}\right) / D, \\
& a_{13}=a_{31}=K_{2} \alpha_{s}\left(1-S_{1}\right)\left(A_{2}+K_{1}\right) / D, \\
& a_{22}=K_{1} S_{1}^{2}\left(1-\alpha_{s}\right)\left(K_{2}+\frac{A_{2}}{S_{1}}\right) / D, \\
& a_{23}=a_{32}=K_{1} K_{2} S_{1}\left(1-S_{1}\right)\left(1-\alpha_{s}\right) / D, \\
& a_{33}=K_{2}\left(1-S_{1}\right)^{2}\left(1-\alpha_{s}\right)\left(K_{s}+\frac{A_{2}}{\left(1-S_{1}\right)}\right) / D, \\
& D=K_{1}\left(1-S_{1}\right)+A_{2}+K_{2} S_{1}, \\
& c_{1}=\left(1-\alpha_{s}\right)^{2} S_{1}^{2} \mu_{1} / K K_{r 1}, \\
& c_{2}=\left(1-\alpha_{s}\right)^{2}\left(1-S_{1}\right)^{2} \mu_{2} / K K_{r 2}, \\
& S_{i}=\alpha_{i} /\left(1-\alpha_{s}\right), \quad(i=1,2), \\
& S_{2}=1-S_{1}, \quad A_{2}=\frac{d P_{\text {cap }}}{d S_{1}} S_{1}\left(1-S_{1}\right),
\end{aligned}
$$

$\mathbf{u}_{s}$ and $\mathbf{u}_{i}$ are the displacement vectors in the porous elastic solid and in the fluid of phase $i$ respectively; $\left\langle\rho_{s}\right\rangle$ is the volume averaged density of porous solid, $\left\langle\rho_{i}\right\rangle$ and $\mu_{i}$ are the volume averaged density and viscosity of fluid phase $i$ respectively. $K_{i}, v_{i}$ and $v_{s}$ are the bulk modulus, velocity of fluid phase $i$ and the velocity of solid phase respectively. $\alpha_{s}$ is the volume fraction of the solid phase, $\alpha_{i}$ is the volume fraction for the fluid phase $i, K$ is the intrinsic permeability of the medium and $K_{r i}$ is the relative permeability of fluid phase $i . G_{f r}$ is the shear modulus of the porous solid whereas $K_{f r}$ is the frame or drained bulk modulus. $P_{\text {cap }}=P_{1}^{*}-P_{2}^{*}$, where $P_{1}^{*}$ is the pressure of non-wetting fluid while $P_{2}^{*}$ is that of wetting fluid and $I$ is a unit tensor matrix.

The averaged stress $\left\langle\tau_{s}\right\rangle$, in the solid phase and the averaged pressure $\left\langle\tau_{i}\right\rangle$, in the fluid phase $i$ are 
given by

$$
\begin{aligned}
\left\langle\tau_{s}\right\rangle= & \left(a_{11} \nabla \cdot \mathbf{u}_{s}+a_{12} \nabla \cdot \mathbf{u}_{1}+a_{13} \nabla \cdot \mathbf{u}_{2}\right) I \\
& +G_{f r}\left(\nabla \mathbf{u}_{s}+\left(\nabla \mathbf{u}_{s}\right)^{T}-\frac{2}{3} \nabla \cdot \mathbf{u}_{s} I\right), \\
\left\langle\tau_{i}\right\rangle= & \left(a_{n 1} \nabla \cdot \mathbf{u}_{s}+a_{n 2} \nabla \cdot \mathbf{u}_{1}+a_{n 3} \nabla \cdot \mathbf{u}_{2}\right) I, \\
& (n=2, \text { if } i=1 \text { and } n=3, \text { if } i=2) .
\end{aligned}
$$

The Helmholtz representation of vectors $\mathbf{u}_{s}$ and $\mathbf{u}_{i}$ is given by

$$
\mathbf{u}_{j}=\nabla \phi_{j}+\nabla \times \mathbf{H}_{j}, \quad \nabla \cdot \mathbf{H}_{j}=0, \quad(j=s, i),
$$

where $\phi_{j}$ are the scalar potentials, while $\mathbf{H}_{j}$ are the vector potentials and they satisfy the following wave equations

$$
\nabla^{2} \phi_{j}=\frac{1}{V_{l}^{2}} \frac{\partial^{2} \phi_{j}}{\partial t^{2}}, \quad \nabla^{2} \mathbf{H}_{s}=\frac{1}{V_{4}^{2}} \frac{\partial^{2} \mathbf{H}_{s}}{\partial t^{2}},
$$

where

$$
l=\left\{\begin{array}{lll}
1 & \text { if } & j=s \\
i+1 & \text { if } & j=i
\end{array}\right.
$$

Tomar and Arora (2006) have shown that in an infinite elastic porous medium saturated by two immiscible fluids, there can travel three longitudinal waves propagating at distinct speeds and a transverse wave. The velocities $V_{l}^{2}(l=1,2,3)$ of the three longitudinal waves are given by the roots of equation $Z_{1} V^{6}+Z_{2} V^{4}+Z_{3} V^{2}+Z_{4}=0$ and the velocity of transverse wave is given by $V_{4}^{2}=-Z_{6} / Z_{5}$. The expressions of entities $Z_{1}, Z_{2}, Z_{3}, Z_{4}, Z_{5}$ and $Z_{6}$ are given in Appendix 1 .

Substituting the values of vectors $\mathbf{u}_{s}, \mathbf{u}_{1}$ and $\mathbf{u}_{2}$ from (6) into equations (2)-(3), we obtain

$$
\begin{aligned}
& \left\langle\rho_{1}\right\rangle \frac{\partial^{2} \mathbf{H}_{1}}{\partial t^{2}}=c_{1}\left(\frac{\partial \mathbf{H}_{s}}{\partial t}-\frac{\partial \mathbf{H}_{1}}{\partial t}\right), \\
& \left\langle\rho_{2}\right\rangle \frac{\partial^{2} \mathbf{H}_{2}}{\partial t^{2}}=c_{2}\left(\frac{\partial \mathbf{H}_{s}}{\partial t}-\frac{\partial \mathbf{H}_{2}}{\partial t}\right) .
\end{aligned}
$$

Taking $\mathbf{H}_{j}(x, z, t)=\mathbf{H}_{j}(x, z) \exp (-\imath \omega t)$, we see from (8) and (9) that the potentials $\mathbf{H}_{i}$ and $\mathbf{H}_{s}$ are related to each other through the relation

$$
\mathbf{H}_{i}=\imath c_{i}\left(\imath c_{i}+\left\langle\rho_{i}\right\rangle \omega\right)^{-1} \mathbf{H}_{\mathbf{s}}
$$

Thus, the potentials $\mathbf{H}_{i}$ can be expressed in terms of potential $\mathbf{H}_{s}$ and since the potential $\mathbf{H}_{s}$ satisfies the wave equation mentioned above, therefore the potentials $\mathbf{H}_{i}$ will also satisfy the wave equation similar to $(7)_{2}$.

Now, we shall derive the frequency equation for the propagation of waves in a cylindrical bore filled with and without fluid.

\section{Empty cylindrical bore}

We consider an empty circular cylindrical borehole of radius ' $a$ ' extending to infinity in the $z$-direction and embedded in a poroelastic medium saturated by two immiscible fluids. We shall seek the propagation of axially symmetric waves at the surface of the cylindrical bore. In the cylindrical coordinates $(r, \theta, z)$, we shall take $\mathbf{u}_{j}=\left(u_{j r}(r, z), 0, u_{j z}(r, z)\right)$ and the displacement vector $\mathbf{u}_{j}$ can be written as

$$
\mathbf{u}_{j}=u_{j r} \mathbf{c}_{r}+u_{j z} \mathbf{c}_{z},
$$

where $\mathbf{c}_{r}$ and $\mathbf{c}_{z}$ are the unit base vectors in the increasing direction of $r$ and $z$ respectively. Let $\mathbf{H}_{j}=H_{j \theta} \mathbf{c}_{\theta}$, where $H_{j \theta}$ is independent of $\theta$ and $\mathbf{c}_{\theta}$ is a unit base vector in the increasing direction of $\theta$. From equations (6) and (7), we get

$$
\begin{aligned}
\mathbf{u}_{j} & =\nabla \phi_{j}+\nabla \times H_{j \theta} \mathbf{c}_{\theta}, \quad \nabla \cdot \mathbf{H}_{\mathbf{j} \theta} \mathbf{c}_{\theta}=\mathbf{0} \\
\nabla & \equiv \frac{\partial}{\partial r} \mathbf{c}_{r}+\frac{\partial}{\partial z} \mathbf{c}_{z}
\end{aligned}
$$

and

$$
\begin{aligned}
\nabla^{2} \phi_{j} & =\frac{1}{V_{l}^{2}} \frac{\partial^{2} \phi_{j}}{\partial t^{2}}, \quad \nabla^{2} H_{j \theta}-\frac{H_{j \theta}}{r^{2}}=\frac{1}{V_{4}^{2}} \frac{\partial^{2} H_{j \theta}}{\partial t^{2}} \\
\nabla^{2} & \equiv \frac{1}{r} \frac{\partial}{\partial r}\left(r \frac{\partial}{\partial r}\right)+\frac{\partial^{2}}{\partial z^{2}}
\end{aligned}
$$

Comparing $\mathbf{u}_{j}$ given in (11) and (12), we obtain

$$
\begin{aligned}
& u_{j r}=\frac{\partial \phi_{j}}{\partial r}-\frac{\partial H_{j \theta}}{\partial z} \\
& u_{j z}=\frac{\partial \phi_{j}}{\partial z}+\frac{H_{j \theta}}{r}+\frac{\partial H_{j \theta}}{\partial r} \quad(j=s, i) .
\end{aligned}
$$


Using equations (4) and (5), the components of stress, $\left\langle\tau_{r r}\right\rangle$ and $\left\langle\tau_{z r}\right\rangle$ in the porous solid phase and the pressure $\left\langle\tau_{i}\right\rangle$ in the porous fluid phase $i$, in terms of potentials $\phi_{j}$ and $H_{j \theta}$ are given by

$$
\begin{aligned}
\left\langle\tau_{r r}\right\rangle= & 2 G_{f r} \frac{\partial^{2} \phi_{s}}{\partial r^{2}}+\frac{\left(a_{11}-\frac{2}{3} G_{f r}\right)}{V_{1}^{2}} \frac{\partial^{2} \phi_{s}}{\partial t^{2}} \\
& +\frac{a_{12}}{V_{2}^{2}} \frac{\partial^{2} \phi_{1}}{\partial t^{2}}+\frac{a_{13}}{V_{3}^{2}} \frac{\partial^{2} \phi_{2}}{\partial t^{2}}-2 G_{f r} \frac{\partial^{2} H_{s \theta}}{\partial r \partial z} \\
\left\langle\tau_{z r}\right\rangle= & 4 G_{f r}\left(\frac{\partial^{2} \phi_{s}}{\partial z \partial r}-\frac{\partial^{2} H_{s \theta}}{\partial z^{2}}\right)+\frac{2 G_{f r}}{V_{4}^{2}} \frac{\partial^{2} H_{s \theta}}{\partial t^{2}}, \\
\left\langle\tau_{1}\right\rangle= & \frac{a_{21}}{V_{1}^{2}} \frac{\partial^{2} \phi_{s}}{\partial t^{2}}+\frac{a_{22}}{V_{2}^{2}} \frac{\partial^{2} \phi_{1}}{\partial t^{2}}+\frac{a_{23}}{V_{3}^{2}} \frac{\partial^{2} \phi_{2}}{\partial t^{2}} \\
\left\langle\tau_{2}\right\rangle= & \frac{a_{31}}{V_{1}^{2}} \frac{\partial^{2} \phi_{s}}{\partial t^{2}}+\frac{a_{32}}{V_{2}^{2}} \frac{\partial^{2} \phi_{1}}{\partial t^{2}}+\frac{a_{33}}{V_{3}^{2}} \frac{\partial^{2} \phi_{2}}{\partial t^{2}} .
\end{aligned}
$$

The appropriate boundary conditions for the propagation of waves in an empty cylindrical bore are vanishing of stresses on the surface of the cylindrical bore of radius ' $a$ '. Mathematically, these boundary conditions are

$$
\tau_{r r}=0, \quad \tau_{z r}=0, \quad \tau_{1}=0, \quad \tau_{2}=0, \quad \text { at } r=a .
$$

Solutions of wave equations in (13) for wave propagating in the $z$-direction can be taken as

$$
\begin{gathered}
\left\{\phi_{s}, \phi_{1}, \phi_{2}, H_{s \theta}\right\}=\left\{A_{1} K_{0}\left(m_{1} r\right), A_{2} K_{0}\left(m_{2} r\right),\right. \\
\left.A_{3} K_{0}\left(m_{3} r\right), A_{4} K_{1}\left(m_{4} r\right)\right\} \exp \imath(k z-\omega t),
\end{gathered}
$$

where $A_{n}(n=1,2,3,4)$ are constants, $k$ is the wavenumber, $\omega$ is the angular frequency and $K_{0}($ ) and $K_{1}()$ are the modified Bessel functions of the second kind and of order zero and one respectively. And

$$
m_{n}=k \sqrt{1-\xi_{n}^{2}}, \quad \xi_{n}=\frac{c}{V_{n}}
$$

where $c$ is the phase velocity of the waves in the $z$ direction. Plugging (20) in the boundary conditions given in (19) and making use of the identities

$$
K_{0}^{\prime}(x)=-K_{1}(x), \quad K_{1}^{\prime}(x)=K_{0}(x)-\frac{1}{x} K_{1}(x),
$$

$$
K_{0}^{\prime \prime}(x)=-K_{0}(x)+\frac{1}{x} K_{1}(x),
$$

we get four homogeneous equations in four unknown, namely $A_{1}, A_{2}, A_{3}$ and $A_{4}$. The condition for non-trivial solution of these unknown yields

$$
\operatorname{det}\left[b_{i j}\right]=0,
$$

where the elements of $4 \times 4$ square matrix $\left\{b_{i j}\right\}$ are zero except for

$$
\begin{aligned}
& b_{11}=\left(2-\xi_{4}^{2}\right) \frac{K_{0}\left(m_{1} a\right)}{K_{1}\left(m_{1} a\right)}+\frac{2}{k a} \sqrt{1-\xi_{1}^{2}}, \\
& b_{12}=-\frac{a_{12}}{G_{f r}} \xi_{2}^{2} \frac{K_{0}\left(m_{2} a\right)}{K_{1}\left(m_{2} a\right)} \\
& b_{13}=-\frac{a_{13}}{G_{f r}} \xi_{3}^{2} \frac{K_{0}\left(m_{3} a\right)}{K_{1}\left(m_{3} a\right)} \\
& b_{14}=-2\left\{\frac{1}{k a}+\sqrt{1-\xi_{4}^{2}} \frac{K_{0}\left(m_{4} a\right)}{K_{1}\left(m_{4} a\right)}\right\} \\
& b_{21}=2 \sqrt{1-\xi_{1}^{2}}, \\
& b_{31}=\frac{a_{21}}{G_{f r}} \xi_{1}^{2} \frac{K_{0}\left(m_{1} a\right)}{K_{1}\left(m_{1} a\right)}, \\
& b_{32}=\frac{a_{22}}{G_{f r}} \xi_{2}^{2} \frac{K_{0}\left(m_{2} a\right)}{K_{1}\left(m_{2} a\right)}, \\
& b_{33}=\frac{a_{23}}{G_{f r}} \xi_{3}^{2} \frac{K_{0}\left(m_{3} a\right)}{K_{1}\left(m_{3} a\right)} \\
& b_{43}=\frac{a_{33}}{G_{f r}} \xi_{3}^{2} \frac{K_{0}\left(m_{3} a\right)}{K_{1}\left(m_{3} a\right)} \\
& b_{41}=\frac{a_{31}}{G_{f r}} \xi_{1}^{2} \frac{K_{0}\left(m_{1} a\right)}{K_{1}\left(m_{1} a\right)} \\
& a_{f r} \\
& \xi_{2}^{2} \frac{K_{0}\left(m_{2} a\right)}{K_{1}\left(m_{2} a\right)}
\end{aligned}
$$

where $\xi_{4}^{2}=\left(\left(a_{11} / G_{f r}\right)+(4 / 3)\right) \xi_{1}^{2}$.

Equation (23) is the frequency equation for the propagation of surface waves at the surface of an empty cylindrical bore. This equation determines the phase velocity of the axially symmetric waves as a function of wavenumber $k a$ and hence these waves are dispersive in nature. For very short wavelengths, i.e, for large value of $k a$, the host medium behaves like a semi-infinite medium and the dispersion equation (23) must converge to the dispersion 
equation of Rayleigh-type surface wave at the plane boundary of a porous solid half-space saturated by two immiscible fluids. For this purpose, making $k a \rightarrow \infty$ in equation (23) and making use of the asymptotic expressions of the modified Bessel function (Abramowitz and Stegun 1965)

$$
K_{0}(u)=K_{1}(u)=\exp (-u) \sqrt{\frac{\pi}{2 u}}, \quad(u=\infty),
$$

we obtain

$$
\begin{gathered}
\left(2-\xi_{4}^{2}\right)\left[\left(2-\xi_{4}^{2}\right)+\left(\frac{a_{12}}{G_{f r}} F_{1}+\frac{a_{13}}{G_{f r}} F_{2}\right) \xi_{1}^{2}\right] \\
=4 \sqrt{1-\xi_{1}^{2}} \sqrt{1-\xi_{4}^{2}}
\end{gathered}
$$

where

$$
F_{1}=\frac{a_{21} a_{33}-a_{23} a_{31}}{a_{22} a_{33}-a_{23} a_{32}}
$$

and

$$
F_{2}=\frac{a_{22} a_{31}-a_{21} a_{32}}{a_{22} a_{33}-a_{23} a_{32}} .
$$

Equation (24) is the dispersion equation of Rayleigh type wave at the free surface of a porous elastic half-space saturated by two immiscible fluids.

Conditions given in (19) are open pore conditions, which do not ensure the restriction of flow of liquids into the borehole. If the borehole is empty, we must ensure the restriction on the interstitial liquid to enter into the borehole. For this, the appropriate boundary conditions are

$\tau_{r r}=0, \quad \tau_{z r}=0, \quad \dot{u}_{s r}-\dot{u}_{1 r}=0, \quad \dot{u}_{s r}-\dot{u}_{2 r}=0$.

These conditions are called closed pore conditions. These conditions are physically realistic as at very high frequency, when porous rock is compressed, the generated pressure of pore-fluids does not have enough time to transmit very far and the rock behaves as if all the surface pores were plugged. Using equations (10), (14), (20) and (22) in the above conditions, we get the corresponding dispersion equation as

$$
\operatorname{det}\left[d_{i j}\right]=0,
$$

where the entries of $4 \times 4$ square matrix $\left\{d_{i j}\right\}$ are zero except for

$$
d_{11}=\left(2-\xi_{4}^{2}\right) \frac{K_{0}\left(m_{1} a\right)}{K_{1}\left(m_{1} a\right)}+2 \sqrt{1-\xi_{1}^{2}} \frac{1}{k a},
$$

$$
\begin{aligned}
& d_{12}=-\frac{a_{12}}{G_{f r}} \xi_{2}^{2} \frac{K_{0}\left(m_{2} a\right)}{K_{1}\left(m_{2} a\right)} \\
& d_{13}=-\frac{a_{13}}{G_{f r}} \xi_{3}^{2} \frac{K_{0}\left(m_{3} a\right)}{K_{1}\left(m_{3} a\right)} \\
& d_{14}=-2\left\{\frac{1}{k a}+\sqrt{1-\xi_{4}^{2}} \frac{K_{0}\left(m_{4} a\right)}{K_{1}\left(m_{4} a\right)}\right\} \\
& d_{21}=2 \sqrt{1-\xi_{1}^{2}}, \quad d_{24}=2-\xi_{4}^{2}, \\
& d_{31}=-\sqrt{1-\xi_{1}^{2}}, \quad d_{32}=\sqrt{1-\xi_{2}^{2}}, \\
& d_{34}=\frac{\left\langle\rho_{1}\right\rangle \omega}{\imath c_{1}+\left\langle\rho_{1}\right\rangle \omega}, \quad d_{41}=-\sqrt{1-\xi_{1}^{2}} \\
& d_{43}=\sqrt{1-\xi_{3}^{2}}, \quad d_{44}=\frac{\left\langle\rho_{2}\right\rangle \omega}{\imath c_{1}+\left\langle\rho_{2}\right\rangle \omega} .
\end{aligned}
$$

Making $k a \rightarrow \infty$, equation (26) reduces to the following equation corresponding to Rayleigh-type surface wave as

$$
\begin{gathered}
\left(2-\xi_{4}^{2}\right)^{2} \sqrt{\frac{1-\xi_{2}^{2}}{1-\xi_{1}^{2}}}+\frac{a_{12}}{G_{f r}} \xi_{2}^{2}\left\{\left(2-\xi_{4}^{2}\right)-2\left(\frac{\left\langle\rho_{1}\right\rangle \omega}{\imath c_{1}+\left\langle\rho_{1}\right\rangle \omega}\right)\right\} \\
+\frac{a_{13}}{G_{f r}} \xi_{3}^{2}\left\{\left(2-\xi_{4}^{2}\right)-2 \sqrt{\frac{1-\xi_{2}^{2}}{1-\xi_{3}^{2}}}\left(\frac{\left\langle\rho_{2}\right\rangle \omega}{\imath c_{2}+\left\langle\rho_{2}\right\rangle \omega}\right)\right\} \\
=4 \sqrt{1-\xi_{2}^{2}} \sqrt{1-\xi_{4}^{2}} .
\end{gathered}
$$

\section{Cylindrical bore filled with a fluid}

Now, we shall derive the frequency equation for the propagation of surface waves in a cylindrical bore filled with a liquid and situated in a porous medium saturated with two immiscible fluids. For the axially symmetric waves, the displacement potential $\phi_{0}$ in the liquid medium satisfies the equation

$$
\nabla^{2} \phi_{0}=\frac{\rho_{0}}{K_{0}} \frac{\partial^{2} \phi_{0}}{\partial t^{2}}, \quad \nabla^{2} \equiv \frac{\partial^{2}}{\partial r^{2}}+\frac{1}{r} \frac{\partial}{\partial r}+\frac{\partial^{2}}{\partial z^{2}},
$$

where $K_{0}$ and $\rho_{0}$ are the Bulk modulus and the density of the liquid respectively. The solution of the equation (28) for the wave propagating in the $z$-direction can be taken as

$$
\phi_{0}=A_{0} I_{0}\left(m_{0} r\right) \exp \imath(k z-\omega t),
$$

where $I_{0}()$ is the modified Bessel function of zero order and the first kind, $m_{0}=k \sqrt{1-\left(c^{2} / v_{0}^{2}\right)}$ and $v_{0}^{2}=\left(K_{0} / \rho_{0}\right)$. 
The pressure $P_{0}$ and the radial displacement $q_{0}$ of the liquid are given by

$$
P_{0}=-\rho_{0} \frac{\partial^{2} \phi_{0}}{\partial t^{2}}=\rho_{0} \omega^{2} A_{0} I_{0}\left(m_{0} r\right) \exp \imath(k z-\omega t),
$$

$$
q_{0}=\frac{\partial \phi_{0}}{\partial r}=-m_{0} A_{0} I_{0}\left(m_{0} r\right) \exp \imath(k z-\omega t)
$$

Following Deresiewicz and Skalak (1963), a possible set of boundary conditions may be taken as

$$
\begin{gathered}
\tau_{r r}=-P_{0}, \quad \tau_{z r}=0, \quad \tau_{1}+\tau_{2}=P_{0}, \\
\dot{u}_{s r}=\dot{q}_{0}, \quad \dot{u}_{1 r}+\dot{u}_{2 r}=\dot{q}_{0} \quad \text { at } r=a .
\end{gathered}
$$

Making use of the equations (10), (15)-(18) and (28)-(31) in the above boundary conditions (32), we obtain five homogeneous equations in five unknowns namely, $A_{0}, A_{1}, A_{2}, A_{3}$ and $A_{4}$. The condition for non-trivial solution of these equations give the corresponding dispersion equation

$$
\operatorname{det}\left[c_{i j}\right]=0 \text {, }
$$

where the nonzero entries of the $5 \times 5$ square matrix $\left\{c_{i j}\right\}$ are given by

$$
\begin{aligned}
& c_{11}=\left(2-\xi_{4}^{2}\right) \frac{K_{0}\left(m_{1} a\right)}{K_{1}\left(m_{1} a\right)}+\frac{2}{k a} \sqrt{1-\xi_{1}^{2}}, \\
& c_{12}=-\frac{a_{12}}{G_{f r}} \xi_{2}^{2} \frac{K_{0}\left(m_{2} a\right)}{K_{1}\left(m_{2} a\right)}, \\
& c_{13}=-\frac{a_{13}}{G_{f r}} \xi_{3}^{2} \frac{K_{0}\left(m_{3} a\right)}{K_{1}\left(m_{3} a\right)}, \\
& c_{14}=-2\left\{\frac{1}{k a}+\sqrt{1-\xi_{4}^{2}} \frac{K_{0}\left(m_{4} a\right)}{K_{1}\left(m_{4} a\right)}\right\}, \\
& c_{15}=\frac{K_{0}}{G_{f r}} \xi_{0}^{2} \frac{I_{0}\left(m_{0} a\right)}{I_{1}\left(m_{0} a\right)}, \\
& c_{21}=2 \sqrt{1-\xi_{1}^{2}}, \\
& c_{31}=\frac{\left(a_{21}+a_{31}\right)}{G_{f r}} \xi_{1}^{2} \frac{K_{0}\left(m_{1} a\right)}{K_{1}\left(m_{1} a\right)}, \\
& c_{32}=\frac{\left(a_{22}+a_{32}\right)}{G_{f r}} \xi_{2}^{2} \frac{K_{0}\left(m_{2} a\right)}{K_{1}\left(m_{2} a\right)},
\end{aligned}
$$

$$
\begin{aligned}
& c_{33}=\frac{\left(a_{23}+a_{33}\right)}{G_{f r}} \xi_{3}^{2} \frac{K_{0}\left(m_{3} a\right)}{K_{1}\left(m_{3} a\right)}, \\
& c_{35}=-\frac{K_{0}}{G_{f r}} \xi_{0}^{2} \frac{I_{0}\left(m_{0} a\right)}{I_{1}\left(m_{0} a\right)}, \\
& c_{41}=-\sqrt{1-\xi_{1}^{2}}, \quad c_{44}=1, \\
& c_{45}=\sqrt{1-\xi_{0}^{2}}, \quad c_{52}=-\sqrt{1-\xi_{2}^{2}}, \\
& c_{53}=-\sqrt{1-\xi_{3}^{2}}, \quad \\
& c_{54}=\left(\frac{\imath c_{1}}{\imath c_{1}+\left\langle\rho_{1}\right\rangle \omega}+\frac{\imath c_{2}}{\imath c_{2}+\left\langle\rho_{2}\right\rangle \omega}\right) \frac{K_{1}\left(m_{4} a\right)}{K_{1}\left(m_{4} a\right)}, \\
& c_{55}=\sqrt{1-\xi_{0}^{2}} .
\end{aligned}
$$

Making $k a \rightarrow \infty$, the equation (33) reduces to

$$
\operatorname{det}\left[f_{i j}\right]=0,
$$

where the nonzero entries of $5 \times 5$ square matrix $\left\{f_{i j}\right\}$ are given by

$$
\begin{array}{ll}
f_{11}=2-\xi_{4}^{2}, & f_{12}=-\frac{a_{12}}{G_{f r}} \xi_{2}^{2}, \\
f_{13}=-\frac{a_{13}}{G_{f r}} \xi_{3}^{2}, & f_{14}=-2 \sqrt{1-\xi_{4}^{2}}, \\
f_{15}=\frac{K_{0}}{G_{f r}} \xi_{0}^{2}, & f_{21}=2 \sqrt{1-\xi_{1}^{2}}, \\
f_{24}=-\left(2-\xi_{4}^{2}\right), & f_{31}=\frac{\left(a_{21}+a_{31}\right)}{G_{f r}} \xi_{1}^{2}, \\
f_{32}=\frac{\left(a_{22}+a_{32}\right)}{G_{f r}} \xi_{2}^{2}, & f_{33}=\frac{\left(a_{23}+a_{33}\right)}{G_{f r}} \xi_{3}^{2}, \\
f_{35}=-\frac{K_{0}}{G_{f r}} \xi_{0}^{2}, & f_{41}=-\sqrt{1-\xi_{1}^{2}}, \\
f_{44}=1, & f_{45}=\sqrt{1-\xi_{0}^{2}}, \\
f_{52}=-\sqrt{1-\xi_{2}^{2}}, & f_{53}=-\sqrt{1-\xi_{3}^{2}}, \\
f_{54}=\frac{\imath c_{1}}{\imath c_{1}+\left\langle\rho_{1}\right\rangle \omega}+\frac{\imath c_{2}}{\imath c_{2}+\left\langle\rho_{2}\right\rangle \omega}, \\
f_{55}=\sqrt{1-\xi_{0}^{2}} .
\end{array}
$$

Equation (34) is the frequency equation for the Rayleigh-type surface waves at the plane interface between a liquid half-space and a porous halfspace saturated by two immiscible fluids. 


\section{Special cases}

- If we neglect the presence of one of the fluids from the host porous medium, then we shall be left with the problem of wave propagation in a cylindrical borehole embedded in a porous medium saturated by a single fluid already discussed by Sharma and Gogna (1990). For this, we neglect the quantities $a_{12}, a_{22}$ and $a_{32}$ corresponding to one of the immiscible fluids, we find that equation (23) reduces to
With the same consideration, the equation (24) reduces to

$$
\left(2-\xi_{4}^{2}\right)^{2}=4 \sqrt{\left(1-\xi_{1}^{2}\right)\left(1-\xi_{4}^{2}\right)} .
$$

This equation does match exactly with the equation (2.17) of Biot (1952) which gives the equation for Rayleigh wave at a plane boundary of a half-space. Equations (36) and (37) exactly match with the equations (2.16) and (3.13) of Biot (1952) for the relevant problems.

$$
\left|\begin{array}{ccc}
\left(2-\xi_{4}^{2}\right) \frac{K_{0}\left(m_{1} a\right)}{K_{1}\left(m_{1} a\right)}+\frac{2}{k a} \sqrt{1-\xi_{1}^{2}} & -\frac{a_{13}}{G_{f r}} \xi_{3}^{2} \frac{K_{0}\left(m_{3} a\right)}{K_{1}\left(m_{3} a\right)} & -2\left\{\frac{1}{k a}+\sqrt{1-\xi_{4}^{2}} \frac{K_{0}\left(m_{4} a\right)}{K_{1}\left(m_{4} a\right)}\right\} \\
2 \sqrt{1-\xi_{1}^{2}} & 0 & -\left(2-\xi_{4}^{2}\right) \\
\frac{a_{31}}{G_{f r}} \xi_{1}^{2} \frac{K_{0}\left(m_{1} a\right)}{K_{1}\left(m_{1} a\right)} & \frac{a_{33}}{G_{f r}} \xi_{3}^{2} \frac{K_{0}\left(m_{3} a\right)}{K_{1}\left(m_{3} a\right)} & 0
\end{array}\right|=0 .
$$

Equation (35) is analogous to the equation (23) of Sharma and Gogna (1990) for the relevant problem.

- If both the fluids are absent from the host porous medium, then the problem reduces to the problem of wave propagation in a cylindrical bore embedded in an elastic medium of infinite extent, already investigated by Biot (1952). To reduce the problem of Biot (1952) from the present formulation, we put all the parameters corresponding to the fluids of the host medium, equal to zero. With $S_{1}=S_{2}=A_{2}=0$, we see that the quantities $a_{12}, a_{22}, a_{32}, a_{13}$ and $a_{33}$ vanish and we find that the frequency equations (23) and (33) corresponding to the empty cylindrical borehole and filled with liquid respectively reduce to

\section{Numerical computations}

Numerical computations are performed to study the behaviour of dispersion curve of surface waves. Equation (23) is solved numerically for a specific model and the following values of relevant elastic parameters for Columbia Fine Sandy Loam saturated by an air-water mixture are taken (Lo et al 2005):

$K_{f r}=8.33 \mathrm{MPa}, K_{s}=35 \mathrm{GPa}, K_{1}=0.145 \mathrm{MPa}$,

$$
K_{2}=2.25 \mathrm{GPa}, \quad G_{f r}=3.85 \mathrm{MPa},
$$

$$
\begin{array}{rr}
\frac{\left(2-\xi_{4}^{2}\right)^{2}}{\sqrt{1-\xi_{1}^{2}}} \frac{K_{0}\left(m_{1} a\right)}{K_{1}\left(m_{1} a\right)}+\frac{2\left(2-\xi_{4}^{2}\right) \sqrt{1-\xi_{1}^{2}}}{m_{1} a}-4\left\{\frac{1}{k a}+\sqrt{1-\xi_{4}^{2}} \frac{K_{0}\left(m_{4} a\right)}{K_{1}\left(m_{4} a\right)}\right\}=0, \\
\left|\begin{array}{ccc}
\left(2-\xi_{4}^{2}\right) \frac{K_{0}\left(m_{1} a\right)}{K_{1}\left(m_{1} a\right)}+\frac{2}{k a} \sqrt{1-\xi_{1}^{2}} & -2\left\{\frac{1}{k a}+\sqrt{1-\xi_{4}^{2}} \frac{K_{0}\left(m_{4} a\right)}{K_{1}\left(m_{4} a\right)}\right\} & \frac{K_{0}}{G_{f r}} \xi_{0}^{2} \frac{I_{0}\left(m_{0} a\right)}{I_{1}\left(m_{0} a\right)} \mid \\
2 \sqrt{1-\xi_{1}^{2}} & -\left(2-\xi_{4}^{2}\right) & 0 \\
-\sqrt{1-\xi_{1}^{2}} & 1 & \sqrt{1-\xi_{0}^{2}}
\end{array}\right|=0 .
\end{array}
$$




$$
\begin{aligned}
& \left\langle\rho_{s}\right\rangle=1457.5 \mathrm{~kg} / \mathrm{m}^{3}, \quad\left\langle\rho_{1}\right\rangle=0.000297 \mathrm{~kg} / \mathrm{m}^{3} \\
& \left\langle\rho_{2}\right\rangle=179.6 \mathrm{~kg} / \mathrm{m}^{3}, \quad c_{1}=c_{2}=0 .
\end{aligned}
$$

Bisection method is used to solve the frequency equation (23) and a FORTRAN Program is developed for this purpose. The non-dimensional phase velocity $c / V_{\min }, V_{\min }=\min \left(V_{1}, V_{2}, V_{3}, V_{4}\right)$ is computed at different values of non-dimensional wavenumber $k a$ varying from 0 to 85 . The results obtained only for fundamental mode of propagation are depicted graphically through figure 1 . This figure shows that the waves along the surface of empty borehole and situated in a porous medium saturated with two immiscible fluids are dispersive. The phase velocity decreases continuously with increasing $k a$ as obtained by Biot (1952). The phase velocity of Rayleigh waves corresponding to the limiting case as $k a \rightarrow \infty$, that is, when wavelength of the waves is very small in comparison to the radius of the cylindrical bore, is also computed from equation (24). The phase velocity of the Rayleigh mode at a free surface of the host half-space is found to be 0.1261 times $V_{\min }$. It is found that as $k a$ takes higher and higher values, the phase velocity of the surface waves approaches to the phase velocity of the Rayleigh waves at a free plane boundary of porous solid saturated by two immiscible fluids. The horizontal line in the figure represents the non-dimensional phase velocity of the Rayleigh mode. The velocity of the Rayleigh mode has also been computed for different values of $\rho_{1}$. By taking the values of $\rho_{1}$ equal to $0.297,0.0297,0.00297$ and 0.000297 in $\mathrm{kg} / \mathrm{m}^{3}$, the velocity of the Rayleigh mode are found to be $0.0616,0.0725,0.0919$ and 0.1261 respectively. Thus, as the density of the first fluid $\left(\left\langle\rho_{1}\right\rangle\right)$ goes on decreasing the phase velocity of the Rayleigh mode increases. So, the density of the two fluids affects the velocity of the Rayleigh mode. We have also computed the group velocity $U$, through the well known formula given by $U=c+k(d c / d k)$. The non-dimensional group velocity $U / V_{\min }$ is computed for various values of non-dimensional wavenumber $k a$. The dispersion curve of group velocity is shown in figure 1 . It is seen that group velocity curve continuously decreases with increase in the wavenumber and at higher values of $k a$, it also approaches to the phase velocity of the Rayleigh mode.

When one of the fluids is neglected from the porous medium, the problem reduces to the problem of propagation of waves along the cylindrical borehole situated in a porous medium saturated by a single fluid. Figure 2 shows the variation of dimensionless phase velocity with the

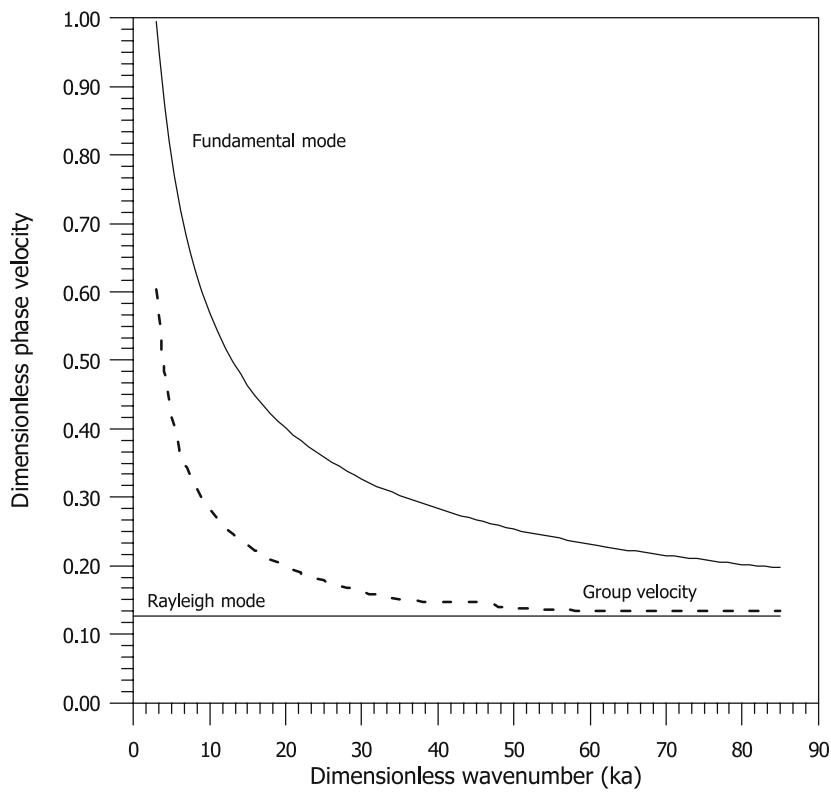

Figure 1. Variation of dimensionless phase velocity and group velocity with dimensionless wavenumber in the case of porous medium saturated by two immiscible fluids.

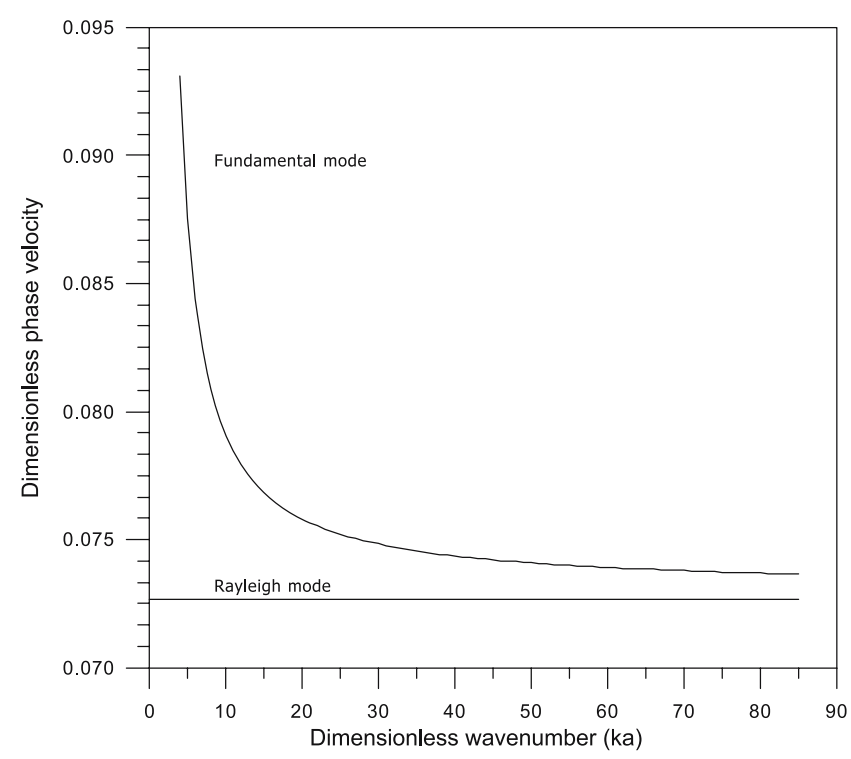

Figure 2. Variation of dimensionless phase velocity with dimensionless wavenumber in the case of porous medium saturated by a single fluid.

dimensionless wavenumber, when porous medium is saturated by a single fluid. In this case, the phase velocity of the Rayleigh wave (horizontal line in figure 2) at free surface of the host half-space is found to be 0.0726 times $V_{\min }$. We also note that at higher values of $k a$, the phase velocity curve becomes asymptotic to the Rayleigh mode value. On comparing the dispersion curves of surface waves in figures 1 and 2, we note that the presence or absence of one of the fluids in the host medium shifts the dispersion curve to a great extent. The amount of 
shift indicates effect of the presence or absence of one of the fluids in the porous medium. It can also be seen that the Rayleigh mode is also influenced greatly by the presence or absence of additional fluid in the host medium.

\section{Acknowledgements}

One of the authors (SKT) is thankful to Council of Scientific and Industrial Research, New Delhi, for providing financial assistance through Grant No. 25 (0134)/04/EMR-II for completing this study. Authors are thankful to the unknown reviewers for their useful suggestions which led to improvement in the manuscript.

\section{Appendix 1}

The expressions of $Z_{1}, Z_{2}, Z_{3}, Z_{4}, Z_{5}$ and $Z_{6}$ are given by

$$
\begin{aligned}
& Z_{1}=\frac{c_{1} c_{2}\left(\left\langle\rho_{1}\right\rangle+\left\langle\rho_{2}\right\rangle+\left\langle\rho_{s}\right\rangle\right)-\left\langle\rho_{s}\right\rangle\left\langle\rho_{1}\right\rangle\left\langle\rho_{2}\right\rangle \omega^{2}}{\omega^{2}} \\
& -\frac{\imath c_{2}\left\langle\rho_{1}\right\rangle\left(\left\langle\rho_{2}\right\rangle+\left\langle\rho_{s}\right\rangle\right)+c_{1}\left\langle\rho_{2}\right\rangle\left(\left\langle\rho_{1}\right\rangle+\left\langle\rho_{s}\right\rangle\right)}{\omega}, \\
& \begin{array}{r}
\left(a_{11}^{*}\left(c_{1} c_{2}-\left\langle\rho_{1}\right\rangle\left\langle\rho_{2}\right\rangle \omega^{2}\right)\right. \\
\left.+2 c_{1} c_{2}\left(a_{12}+a_{13}+a_{23}\right)\right)
\end{array} \\
& \left(a_{22}\left(c_{1} c_{2}-\left\langle\rho_{s}\right\rangle\left\langle\rho_{2}\right\rangle \omega^{2}\right)\right. \\
& -\frac{\left.+a_{33}\left(c_{1} c_{2}-\left\langle\rho_{s}\right\rangle\left\langle\rho_{1}\right\rangle \omega^{2}\right)\right)}{\omega^{2}} \\
& \left(a_{11}^{*}\left(c_{2}\left\langle\rho_{1}\right\rangle+c_{1}\left\langle\rho_{2}\right\rangle\right)\right. \\
& +\imath \frac{\left.+2 a_{12} c_{1}\left\langle\rho_{2}\right\rangle+2 a_{13} c_{2}\left\langle\rho_{1}\right\rangle\right)}{\omega} \\
& \left(a_{22}\left(c_{2}\left(\left\langle\rho_{2}\right\rangle+\left\langle\rho_{s}\right\rangle\right)+c_{1}\left\langle\rho_{2}\right\rangle\right)\right. \\
& +\imath \frac{\left.+a_{33}\left(c_{2}\left\langle\rho_{1}\right\rangle+c_{1}\left(\left\langle\rho_{s}\right\rangle+\left\langle\rho_{1}\right\rangle\right)\right)\right)}{\omega}, \\
& Z_{3}=-a_{11}^{*}\left(a_{22}\left\langle\rho_{2}\right\rangle+a_{33}\left\langle\rho_{1}\right\rangle\right)+a_{12}^{2}\left\langle\rho_{2}\right\rangle+a_{13}^{2}\left\langle\rho_{1}\right\rangle \\
& -\left\langle\rho_{s}\right\rangle\left(a_{22} a_{33}-a_{23}^{2}\right) \\
& \left(a_{11}\left(a_{22} c_{2}+a_{33} c_{1}\right)\right. \\
& -a_{12}^{2} c_{2}-2 a_{12}\left(a_{21} c_{2}-a_{33} c_{1}\right) \\
& -\imath \frac{\left.-a_{13}^{2} c_{1}+2 a_{13}\left(a_{22} c_{2}-a_{23} c_{1}\right)\right)}{\omega}
\end{aligned}
$$

$$
\begin{aligned}
& -\imath \frac{\left(c_{1}+c_{2}\right)\left(a_{22} a_{33}-a_{23}^{2}\right)}{\omega}, \\
Z_{4}= & a_{11}^{*}\left(a_{22} a_{33}-a_{23}^{2}\right)-a_{12}^{2} a_{33} \\
& +a_{13}\left(2 a_{12} a_{23}-a_{13} a_{22}\right), \\
Z_{5}= & \frac{c_{1} c_{2}\left(\left\langle\rho_{1}\right\rangle+\left\langle\rho_{2}\right\rangle+\left\langle\rho_{s}\right\rangle\right)-\left\langle\rho_{s}\right\rangle\left\langle\rho_{1}\right\rangle\left\langle\rho_{2}\right\rangle \omega^{2}}{\omega^{2}} \\
- & \imath \frac{c_{2}\left\langle\rho_{1}\right\rangle\left(\left\langle\rho_{2}\right\rangle+\left\langle\rho_{s}\right\rangle\right)+c_{1}\left\langle\rho_{2}\right\rangle\left(\left\langle\rho_{1}\right\rangle+\left\langle\rho_{s}\right\rangle\right)}{\omega}, \\
Z_{6}= & \frac{-G_{f r}\left(c_{1} c_{2}-\left\langle\rho_{1}\right\rangle\left\langle\rho_{2}\right\rangle \omega^{2}\right)}{\omega^{2}} \\
& +\imath \frac{G_{f r}\left(c_{2}\left\langle\rho_{1}\right\rangle+c_{1}\left\langle\rho_{2}\right\rangle\right)}{\omega} .
\end{aligned}
$$

\section{References}

Abramowitz M and Stegun I A 1965 Handbook of Mathematical Functions (New York: Dover).

Berger J R, Martin P A and McCaffery S J 2000 Time harmonic torsional waves in a composite cylinder with an imperfect interface; J. Acoust. Soc. Am. 107(3) 1161-1167.

Biot M A 1952 Propagation of elastic waves in a cylindrical borehole containing a fluid; J. Appl. Phys. 23 997-1005.

Cheng C H, Jinzhong Z and Burns D R 1987 Effects of in situ permeability on the propagation of Stoneley (tube) waves in a borehole; Geophysics 52(9) 1279-1289.

Cheng C H and Toksoz M N 1981 Elastic wave propagation in a fluid filled borehole and synthetic acoustic logs; Geophysics 46 1042-1053.

Deresiewicz H and Skalak R 1963 On uniqueness in dynamic poroelasticity; Bull. Seismol. Soc. Am. 53 783-789.

Kurkjian A L and Chang Shu-Kong 1986 Acoustic multipoles in fluid-filled boreholes; Geophysics 51 148-163.

Lo Wei-Cheng, Sposito G and Majer E 2005 Wave Propagation through elastic porous media containing two immiscible fluids; Water Resour. Res. 41 WO2025 doi: 10.1029/200-4WR003162.

Paillet F L and Cheng C H 1986 A numerical investigation of head waves and leaky modes in fluid filled boreholes; Geophysics 51(7) 1438-1449.

Paillet F L and White J E 1982 Acoustic modes of propagation in the borehole and their relationship to rock properties; Geophysics $\mathbf{4 7} 1215-1228$.

Rama Rao V N and Vandiver J K 1999 Acoustics of fluidfilled boreholes with pipe: Guided propagation and radiation; J. Acoust. Soc. Am. 105(6) 3057-3066.

Rosenbaum J H 1974 Synthetic micro-seismograms: logging in porous formations; Geophysics 39 14-32.

Sharma M D and Gogna M L 1990 Propagation of elastic waves in a cylindrical bore in a liquid saturated porous solid; Geophys. J. Int. 103 47-54.

Stilke G 1959 On elastic surface waves at a cylindrical hole in an infinite solid; Geophysical Prospecting 7(3) 273-286. 
Tomar S K and Arora A 2006 Reflection and transmission of elastic waves at an elastic/porous solid saturated by two immiscible fluids; Int. J. Solid. Struct. 43 1991-2013.

Tomar S K and Kumar R 1999 Elastic wave propagation in a cylindrical bore situated in a micropolar elastic medium with stretch; Proc. Indian Acad. Sci. (Math. Sci.) 109(4) 425-433.
Tuncay K and Corapcioglu M Y 1997 Wave propagation in poroelastic media saturated by two fluids; J. Appl. Mech. 64 313-319.

Vashishth A K and Khurana P 2005 Wave propagation along a cylindrical borehole in an anisotropic poroelastic solid; Geophys. J. Int. 161 295-302.

White J E 1962 Elastic waves along a cylindrical bore; Geophysics 27(3) 327-333.

MS received 19 May 2006; revised 30 October 2006; accepted 17 January 2007 